\title{
Editorial
}

\section{Mechanisms of fetal tachycardia}

Fetal tachycardia is an important cause of fetal morbidity and mortality. ${ }^{12}$ Reliable diagnosis in utero has been possible only since the introduction of ultrasound examination of the fetal heart. ${ }^{2}$ In the early days little thought was given to the mechanism of tachycardia, and treatment involved maternal administration of drugs fashionable at the time for treating supraventricular tachycardia in infants and children. Drug treatment was often followed by a return to sinus rhythm, which was interpreted as a response to treatment. However, the natural history of fetal tachycardia was, and remains, unknown.

Resolution of tachycardia, sometimes many days after institution of treatment, may be spontaneous rather than a response to treatment. Digoxin was the mainstay of treatment and verapamil was often the second choice. Both of these drugs exert their main effect on the atrioventricular node. Some deaths occurred with treatment and it was not clear whether this was because treatment was ineffective or the drugs actually caused the death. Most antiarrhythmic drugs have a negative inotropic effect that might be poorly tolerated by the failing fetal myocardium. Digoxin has relatively little effect in suppressing infant supraventricular tachycardia ${ }^{45}$ and intravenous verapamil is recognised as potentially hazardous in early infancy. ${ }^{6}$ The introduction of newer antiarrhythmic drugs, such as amiodarone and flecainide, which exert their main effects on arrhythmia substrates other than the atrioventricular node, led to an increased efficacy of treatment and a reduction in mortality. ${ }^{7-9}$ However, not all fetuses respond to these drugs. It is appropriate to consider in more detail the likely mechanism of fetal tachycardia and the way in which identification of the mechanism might affect the choice of treatment.

\section{Mechanisms of supraventricular tachycardia in infancy and childhood}

The term supraventricular tachycardia covers a wide variety of tachycardia mechanisms. Many are caused by reentry and others are automatic; some are confined to the atria and others involve the atria and the ventricles. The introduction of drugs with more specific actions and, more recently, of radiofrequency ablation, has focused attention on the mechanism of supraventricular tachycardia. It is no longer appropriate to reach a diagnosis of supraventricular tachycardia without consideration or identification of the mechanism and the substrate.

The spectrum of causes of supraventricular tachycardia changes throughout childhood. ${ }^{10}$ In early infancy most supraventricular tachycardia is orthodromic atrioventricular reentry via an accessory pathway. This arrhythmia probably accounts for about $90 \%$ of cases, ${ }^{411}$ but other arrhythmias do occur: atrial flutter, chaotic or multifocal atrial tachycardia, atrial ectopic tachycardia, permanent junctional reciprocating tachycardia, and His bundle tachycardia (junctional ectopic tachycardia). The last three of these are often incessant and characteristically show a poor response to antiarrhythmic drug treatment.

Identification of the mechanism is usually possible from the electrocardiogram as the features of most of these arrhythmias are distinctive. ${ }^{12}$ Analysis involves identification of the $\mathrm{P}$ waves and their relation to QRS complexes.
Thus tachycardia persisting in the face of atrioventricular block (spontaneous or induced by adenosine) will lead to a diagnosis of atrial tachycardia and will exclude atrioventricular reentry. Elucidation of the mechanism of a tachycardia with a 1:1 atrioventricular relation involves consideration of the timing of the $\mathrm{P}$ wave. A short RP interval is characteristic of an accessory pathway, simultaneous atrial and ventricular activation (usually with $P$ waves not visible) occurs in atrioventricular nodal reentry tachycardia, while some arrhythmias are characterised by a long RP interval, particularly atrial ectopic tachycardia and permanent junctional reciprocating tachycardia. ${ }^{12}$ His bundle tachycardia usually shows ventriculoatrial dissociation with slower and dissociated $\mathrm{P}$ waves.

\section{Indirect evidence of the mechanism of fetal tachycardia}

Retrospective evidence for the mechanism and substrate of fetal arrhythmias can be obtained postnatally in survivors by observation of spontaneous recurrences or by transoesophageal electrophysiology studies. Such investigations confirm that the spectrum of electrophysiological abnormalities in those presenting prenatally is similar to that seen in babies who present postnatally. ${ }^{11}{ }^{13}$ Orthodromic atrioventricular reentry accounts for the majority but atrial ectopic tachycardia and other atrial arrhythmias are found.

\section{Direct evidence of the mechanism of fetal tachycardia}

The mechanisms of tachycardia occurring prenatally are likely to be similar to those presenting postnatally. Although previous reports have failed to differentiate or investigate the underlying cause of tachycardia, most separate atrial flutter (very rapid atrial rate with atrioventricular block) from supraventricular tachycardia (with a 1:1 atrioventricular relation). Knowledge of the mechanism of tachycardia is potentially important as it will define both the response to treatment and prognosis.

The variable outcome of individual fetuses with supraventricular tachycardia might be explained by different arrhythmia mechanisms rather than variation in response of the same arrhythmia to drug treatment. Whether the rate of sustained fetal tachycardia with a $1: 1$ atrioventricular relation will help define the mechanism is unknown. In the neonate, orthodromic atrioventricular reentry is usually faster than atrial ectopic tachycardia or permanent junctional reciprocating tachycardia, but in the fetus most tachycardias occur at about 240 beats/min.

In this issue, Jaeggi et al report an investigation of fetal tachycardia that analysed the timing of the ventriculoatrial relations using $\mathrm{M}$ mode echocardiography. ${ }^{15}$ They found that $83 \%$ (95\% confidence interval (CI) 61 to 95) of cases had short ventriculoatrial times characteristic of atrioventricular reentry with the common type of accessory pathway. In $17 \%$ (95\% CI 5 to 39) of cases there was a long ventriculoatrial time typical of permanent junctional reentry tachycardia or atrial ectopic tachycardia. In the latter group, three of four babies were born alive and the diagnosis was confirmed as permanent junctional reentry tachycardia in two cases and atrial ectopic tachycardia in one. 


\section{Implications for treatment}

There is good evidence that most fetal tachycardias are caused by atrioventricular reentry via the common type of accessory pathway. Maternal digoxin remains the drug of first choice in many centres but, in the absence of any knowledge of the natural history, evaluation of the efficacy of this treatment is impossible. Fetal tachycardia associated with hydrops has a poorer outlook and in this situation digoxin is known to be less effective. Flecainide or amiodarone are usually used in the presence of hydrops with an improvement in outcome. Failure to respond to treatment may be an indication for the use of other drugs, but should also raise the possibility that the arrhythmia mechanism is one of the less common, more incessant, and more drug refractory types of supraventricular tachycardia.

Department of Paediatric Cardiology,

Freeman Hospital,

Newcastle upon Tyne NE7 7DN, UK

CHRISTOPHER WREN

1 Van Engelen AD, Weijtens O, Brenner JI, et al. Management outcome and follow-up of fetal tachycardia. I Am Coll Cardiol 1994:24:1371-5.

2 Maxwell DJ, Crawford DC, Curry PVM, et al. Obstetric importance, diagnosis, and management of fetal tachycardias. BMF 1988:297:107-10.
3 Kleinman CS, Copel JA, Weinstein EM, et al. In utero diagnosis and treatment of fetal supraventricular tachycardia. Semin Perinatol 1985;9:113-29.

4 Benson DW, Dunningan A, Benditt DG, et al. Prediction of digoxin treatment failure in infants with supraventricular tachycardia: role of transesophageal pacing. Pediatrics 1985:75:288-93.

5 O'Sullivan JJ, Gardiner HM, Wren C. Digoxin or flecainide for prophylaxis of supraventricular tachycardia in infants. F Am Coll Cardiol 1995:26:9914.

6 Garson A Jr. Medicolegal problems in the management of cardiac arrhythmias in children. Pediatrics $1987 ; 79: 84-8$.

7 Frohn-Mulder IM, Stewart PA, Witsenburg M, et al. The efficacy of flecainide versus digoxin in the management of fetal supraventricular nide versus digoxin in the management
tachycardia. Prenat Diagn 1995;15:1209-13.

8 Meijboom EJ, Van Engelen AD, Van de Beek EW, et al. Fetal arrhythmias. Curr Opin Cardiol 1994:9:97-102.

9 Allan LD, Chita SK, Sharland GK, et al. Flecainide in the treatment of fetal tachycardias. Br Heart f 1991:65:46-8.

10 Ko JK, Deal BJ, Strasburger JF, et al. Supraventricular tachycardia mechanisms and their age distribution in pediatric patients. Am f Cardiol 1992;69:1028-32.

11 Weindling SN, Saul JP, Walsh EP. Efficacy and risks of medical therapy for supraventricular tachycardia in neonates and infants. Am Heart $\mathcal{f}$ 1996:131:66-72.

12 Saul JP, Walsh EP, Triedman JK. Mechanisms and therapy of complex arrhythmias in pediatric patients. F Cardiovasc Electrophysiol 1995:6:112948

13 Zales VR, Dunningan A, Benson DW. Clinical and electrophysiologic features of fetal and neonatal paroxysmal atrial tachycardia resulting in congestive heart failure. Am $\mathcal{F}$ Cardiol 1988:62:225-8.

14 Naheed ZJ, Strasburger JF, Deal BJ, et al. Fetal tachycardia: mechanisms and predictors of hydrops fetalis. $\mathcal{F}$ Am Coll Cardiol 1996:27:1736-40.

15 Jaeggi E, Fouron JC, Fournier A, et al. Ventriculo-atrial time measured on M mode echocardiography: a determining element in diagnosis, treatment, and prognosis of fetal supraventricular tachycardia. Heart 1998;79:582-7. 